\title{
The Protective Effect of a Persistent Trigeminal Artery on Brain Stem Infarctions: A Follow-up Case Report
}

\author{
Yasuhiro Ito*,**, Hirohisa Watanabe**, Hisayoshi Niwa*, Shigetaka HaKusui**, \\ Tetsuo ANDo**, Takeshi YasudA*, Gen SobuE* and Tsutomu Yanagi**
}

\begin{abstract}
A persistent trigeminal artery (PTA) represents an embryonic vascular anastomosis connecting the carotid and basilar arterial systems. Little is known about its protective role in cases of basilar artery occlusion. We followed up a 63-year-old man who had suffered a brain stem infarction due to basilar artery stenosis and was found to have a PTA. Although a second brain stem infarction due to basilar artery occlusion developed, the circulation to the brain stem was well maintained via collateral flow from the PTA, and the patient demonstrated good recovery. A PTA may function as an anastomosis between the carotid and basilar systems, thus preventing a more serious infarction.
\end{abstract}

(Internal Medicine 37: 334-337, 1998)

Key words: basilar artery occlusion, good recovery, collateral circulation, cerebral angiography

\section{Introduction}

A persistent trigeminal artery (PTA) has been found in $0.02 \%$ to $1.25 \%$ of patients undergoing cerebral angiography $(1-4)$ and represents a rare vascular anastomosis between the carotid and basilar arterial systems. In the 3- to 14-mm embryonic stage, the trigeminal artery normally exists and functions to provide blood flow to the primitive hindbrain. During subsequent embryonic development, the trigeminal artery involutes and disappears with the formation of the basilar system, including the circle of Willis and the vertebrobasilar arteries (5). When a trigeminal artery persists into adult life, it is frequently reported to cause ischemic cerebrovascular accidents in the posterior regions of the brain stem and occipital area $(1-4,6-$ 9 ). A PTA is further suspected of correlating with the presence of other vascular disorders such as aneurysms (2), Moyamoya disease (10), tic doulourex (11), and cranial nerve palsy (12). In other cases, a PTA is found only by chance (1). However, there has been little documentation on the protective role of a PTA in occlusive arterial diseases. Here, we now report a patient who exhibited a good recovery from brain stem infarction with basilar artery occlusion, presumably saving his blood supply from the internal carotid artery through a PTA.

\section{Case Report}

A 63-year-old diabetic man had no neurologic symptoms until the morning of February 2, 1991, when he experienced double vision, dysarthria and a right hemiparesis. Neurologic examination on admission revealed a left facial palsy, right hemiparesis and left medial longitudinal fasciculus syndrome. General physical examination revealed no other abnormalities; the blood pressure was normal $(140 / 84 \mathrm{mmHg})$, and the heart rate regular (76 beats per minute). Laboratory findings on admission showed that his plasma glucose level was $198 \mathrm{mg} / \mathrm{dl}$, hemoglobin A1c 7.8\%, total cholesterol $176 \mathrm{mg} / \mathrm{dl}$, and triglyceride $196 \mathrm{mg} / \mathrm{dl}$.

Neuroradiologically, magnetic resonance (MR) imaging detected a high intensity area on the left side of the pons on T2weighted image. The lesion extended to the basal surface of the pons. Cerebral angiography revealed about an $80 \%$ stenosis in the middle portion of the basilar artery (BA) (Fig. 1). Through the stenosis, however, anterograde flow was present, and both distal portions of BA and posterior cerebral arteries (PCAs) were visualized. Left and right vertebral arteries were slightly asymmetrical in size but not hypoplastic. Left carotid angiography demonstrated no stenotic area but revealed a large PTA. The brain stem infarction in this patient was thought to be due to atherosclerotic stenosis of the BA involving the paramedian

From *the Department of Neurology, Nagoya University School of Medicine, Nagoya and **the Department of Neurology, Nagoya Daini Red Cross Hospital, Nagoya

Received for publication April 25, 1997; Accepted for publication November 20, 1997

Reprint requests should be addressed to Dr. Yasuhiro Ito, the Department of Neurology, Nagoya University School of Medicine, 65 Tsurumai-cho, Showa-ku, Nagoya 466-8550 


\section{Persistent Trigeminal Artery}

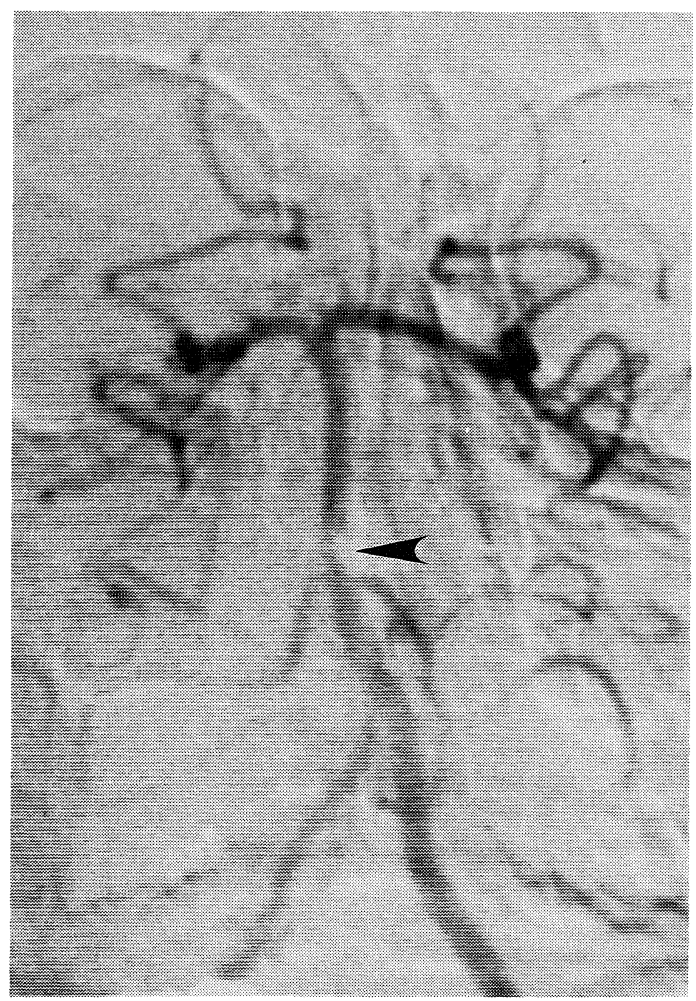

Figure 1. Vertebrobasilar angiography showing an $80 \%$ basilar artery stenosis at the middle portion (arrowhead) of the vessels at the time of the first brain stem infarction.

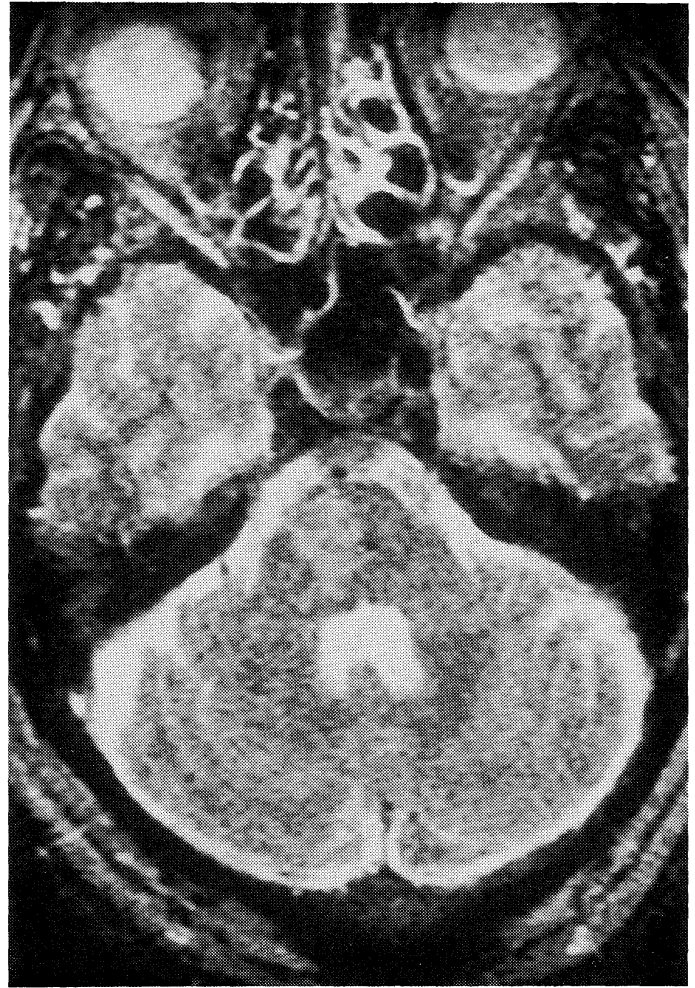

Figure 2. MR T2-weighted image showing a wedge-shaped infarction extending to the basal surface.

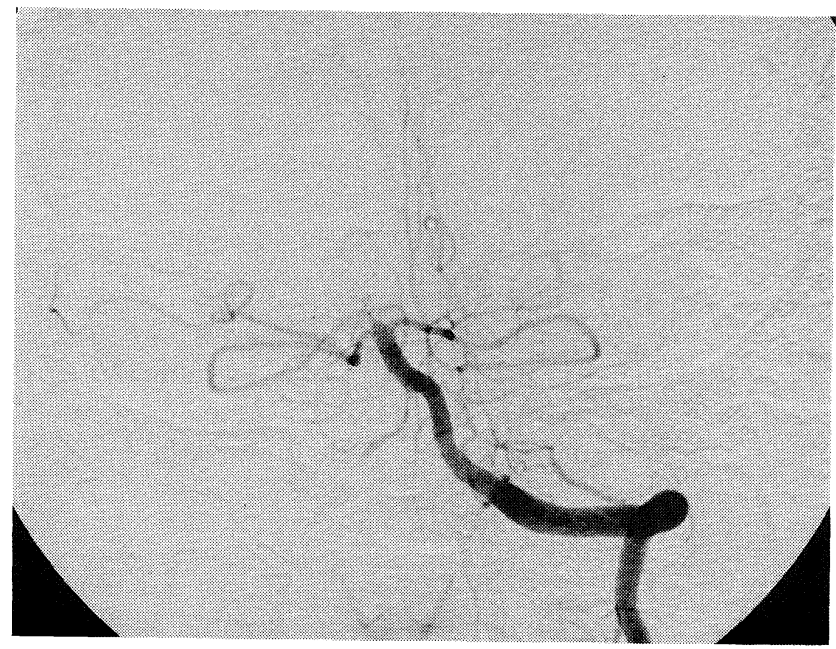

Figure 3. Vertebrobasilar angiography approximately 30 days later at the time of the second brain stem infarction, revealing complete occlusion of the basilar artery.

angiography then showed that complete occlusion of the BA at its middle lower portion. Despite emergency intraarterial fibrinolysis therapy with $960,000 \mathrm{IU}$ urokinase, the BA occlusion persisted. However, his level of consciousness had completely cleared by the next morning, and the hemiparesis and hemihypesthesia were also resolved within a few days. MR imaging demonstrated new T2 high-intensity and T1 lowface. This new lesion was located at the opposite and slightly rostral portion of the first infarction and was thought to present brain stem infarction (Fig. 2). intensity areas in the right side of the pons extending to the basal 


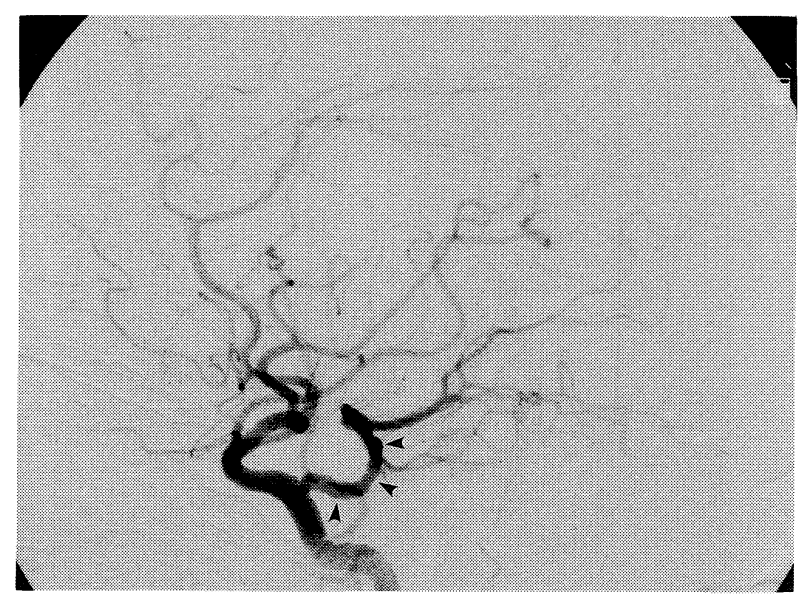

A

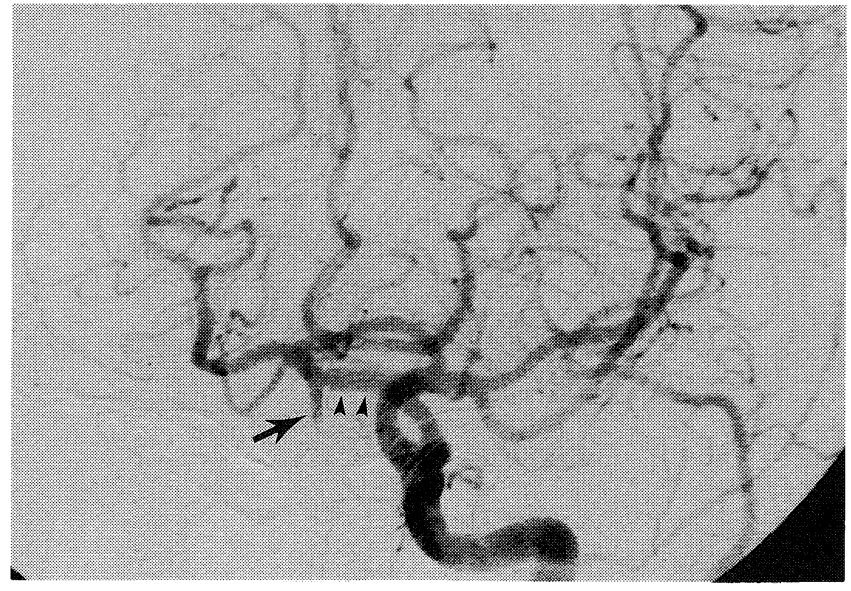

B

Figure 4. A) Left carotid angiography showing a PTA and the posterior circulation. B) The distal portion of the occluded basilar artery (arrow) is visible through a PTA by retrograde flow. Arrowheads indicate a PTA.

There were no other infarctions in the posterior or carotid circulation territory. Cerebral angiography again on the 30th hospital day revealed a complete BA occlusion at its middle lower portion above the level of the anterior inferior cerebellar arteries (AICAs) with no change upon the occurrence of the second stroke (Fig. 3). Left carotid angiography revealed that the distal portion of the occluded BA, superior cerebellar arteries (SCAs) and PCAs of both sides were detectable through a PTA (Fig. 4). The size of the PTA did not change after the BA occlusion. However, a retrograde flow was newly recognized at the distal portion of the BA. The posterior communicating artery (PCOM) was not visualized. Further angiographic examination including aortography showed no stenotic lesions in the bilateral vertebral or carotid arteries. Cardiac studies including electrocardiogram, transthoracic echocardiogram, and Holter monitoring revealed no abnormality. It was considered that the atherosclerotic stenosis of the BA recognized at the first stroke episode became completely occluded. The patient was discharged and was able to walk unassisted. For more than four years after the second cerebrovascular episode, there has been no recurrence, and he is able to perform normal daily activities without any assistance.

\section{Discussion}

A PTA represents a rare embryonic vascular anastomosis between the carotid and basilar arterial systems. When a trigeminal artery persists into adult life, it is frequently suspected that a hypoplastic or anaplastic vertebrobasilar system has developed with an eventual reduction in the blood supply to the brain stem (6). Furthermore, when the carotid artery becomes stenosed due to atherosclerosis, the steal phenomenon from the basilar to the carotid circulation through a PTA may occur, causing vertebrovasilar insufficiency and/or a hemodynamic brain stem infarction (8). The present patient, however, had no hypoplastic or anaplastic vertebrobasilar arteries and no stenotic areas have been recognized in the carotid territory.

In our patient, both the first and second brain stem infarctions extended to the basal surface of the brain stem. This type of infarction is considered to be due to the occlusion at the mouth of the perforating branch, and the etiological mechanism of the infarct is heterogeneous, i.e., embolic or atherosclerotic (1315). Our patient had no cardiological problems presenting a risk of an embolus. No stenotic lesions considered to be the source of artery-to-artery emboli were detected in the vertebrovasilar arterial systems. It is also suspected in a patient with a PTA that the emboli to the posterior area originate in the internal carotid arterial territory and pass through a PTA. However, our patient had no stenotic lesions in the internal carotid arteries $(3,4)$. There is little suspicion that the infarction was due to an embolus.

Atherosclerotic stenosis of a BA occurs frequently at the middle lower portion above the level of the AICAs as recognized in the present patient at the first attack $(16,17)$. He has diabetes mellitus, which is a major risk factor for atherosclerosis of a BA (14). It is considered that the atherosclerotic stenotic lesion became occluded in the second stroke episode.

When a BA occlusion occurs, it is often life-threatening (18), and especially when intra-arterial fibrinolysis therapy is ineffective; the prognosis is generally poor $(19,20)$. Furthermore, in the BA occlusion caused by atherosclerosis, recanalization therapy is less effective $(17,19,20)$. Our case, however exhibited a dramatic recovery. Of course, some cases of BA occlusion with only minor attacks or asymptomatic have been reported (21). Therefore, the severity of occlusive disease involving the posterior circulation varies depending on many factors including the anatomical variation of the vertebrobasilar system, the locus and rapidity of the occlusive process, and the availability of adequate collateral circulation (21). With regard to collateral circulation in cases of BA occlusion, PCOMs or 


\section{Persistent Trigeminal Artery}

leptomeningial anastomosis between middle and posterior cerebral arteries could work as functional collaterals (18). However, in the present case, these anastomoses did not function as collaterals, although the prognosis was extremely favorable with minimal residual neurologic deficits. The retrograde flow of a BA through a PTA was recognized after the second stroke and may have functioned as an effective collateral to the brain stem. There have been no reported follow-up cases in which a PTA provided anterior-posterior collateral circulation following BA occlusion. We, therefore, suggest that the PTA may have played an important role in supplying blood flow to the upper half portion of the brain stem and occipital area following BA occlusion as demonstrated in our patient.

\section{References}

1) Fields WS. The significance of the persistent trigeminal artery: Carotidbasilar anastomosis. Radiology 91: 1096, 1968.

2) Garza-Mercado R, Cavazos E. Persistent trigeminal artery associated with intracranial arterial aneurysm. Neurosurgery 14: 604, 1984.

3) Gasecki AP, Fox AJ, Lebrun LH, Daneault N and The Collaborations of the North American Carotid Endarterectomy Trial (NASCET). Bilateral occipital infarctions associated with carotid stenosis in a patient with persistent trigeminal artery. Stroke 25: 1520, 1994.

4) Khodadad G. Trigeminal artery and occlusive cerebrovascular disease. Stroke 8: 177, 1977.

5) Padget DH. The development of the cranial arteries in the human embryo. Contrib Embryol 32: 205, 1948.

6) Momma F, Ohara S, Ohyama T. Persistent trigeminal artery associated with brain stem infarct-Case report-. Neurol Med Chir (Tokyo) 32: 289, 1992.

7) Waller FT, Simons RL, Kerber C, Kiesel IO, Tanabe CT. Trigeminal artery and microemboli to the brain stem: report of two cases. J Neurosurg 46: 104, 1977.
8) Palmer S, Gücer G. Vertebrobasilar insufficiency from carotid disease associated with a trigeminal artery. Neurosurgery 8: 458, 1981.

9) Quencer RM, Simon J. Transient bilateral occipital lobe ischemia: microembolization through a trigeminal artery. Neuroradiology 18: 273, 1979.

10) Kwak R, Kadoya S. Moyamoya disease associated with persistent primitive trigeminal artery. Reports of two cases. J Neurosurg 59: 166, 1983.

11) Morita A, Fukushima $T$, Miyazaki S, Shimazu, Atsuchi M. Tic douloureux caused by primitive trigeminal artery or its variant. J Neurosurg 70: $415,1989$.

12) Cahill DW, Salcman M, Hirsch D, Rao CV. Unilateral internuclear ophthalmoplegia due to angiographic embolism through a primitive trigeminal artery. Neurology 31: 751, 1981.

13) Caplan LR. Intracranial branch atheromatous disease: a neglected, understudied, and underused concept. Neurology 39: 1246, 1989.

14) Hirai S. Brainstem Infarct - Frequency, Background Factors and Pathogenesis in: Cerebral Stroke in the Era of CT\&MRI, Nihonrinsho, 1993, p. 722 (in Japanese).

15) Toyoda K, Saku Y, Ibayashi S, Sadoshima S, Ogasawara T, Fujishima M. Pontine infarction extending to the basal surface. Stroke 25: 2171, 1994.

16) Peesin MS, Gorelick PB, Kwan ES, Caplan LR. Basilar artery stenosis: Middle and distal segments. Neurology 37: 1742, 1987.

17) Brückmann H, Ferbert A, Del Zoppo GJ, Hacke W, Zeumer H. Acute vertebral-basilar thrombosis: angiologic-clinical comparison and therapeutic implication. Acta Radiol Supple 369: 38, 1987.

18) Caplan LR, Basilar Artery Occlusive Disease. in: Posterior Circulation Disease: Clinical Finding, Diagnosis, and Management, Caplan LR, Eds. Blackwell Science, London, 1996, p. 324.

19) Hacke W, Zeumer H, Ferert A, Brückmann H, Del Zoppo GJ. Intraarterial thrombolytic therapy improves outcome in patients with acute vertebrobasilar occlusive disease. Stroke 19: 1216, 1988.

20) Brandt T, von Kummer R, Müller-Küppers M, Hacke W. Thrombolytic therapy of acute basilar artery occlusion: Variables affecting recanalization and outcome. Stroke 27: 875, 1996.

21) Caplan LR. Occlusion of the vertebral or basilar artery. Follow up analysis of some patients with benign outcome. Stroke 10: 277, 1979. 